\title{
CONSTRUCTION OF SOME SETS OF MUTUALLY ORTHOGONAL LATIN SQUARES
}

\author{
E. T. PARKER
}

H. F. MacNeish [1] demonstrated constructively the existence of a set of $t$ mutually orthogonal latin squares of each order $n$, where $t$ is one less than the smallest factor of the prime-power decomposition of $n$. The construction was generalized somewhat and put on an algebraic foundation by H. B. Mann $[2 ; 3$, p. 105]. MacNeish [1] conjectured that $t$ is the maximum number for each $n$. Had this conjecture been established, answers to two major questions would have been corollaries. These are: (1) the famous conjecture of Euler, dating from 1782, that there exists no pair of orthogonal latin squares of order $\equiv 2(\bmod 4) ;(2)$ the conjecture that all finite projective planes are of prime-power orders - for an affine plane of order $n$ is equivalent to a set of $n-1$ mutually orthogonal latin squares of order $n$.

The purpose of this paper is to develop a construction yièlding some new sets of mutually orthogonal latin squares. The general result is Theorem 1. For a few orders (possibly infinitely many distributed sparsely among the positive integers), Theorem 2 establishes the existence of sets of more than $t$ mutually orthogonal latin squares; thus MacNeish's conjecture is disproved. Theorem 1 likely yields more than $t$ for orders other than those covered by Theorem 2, but the author has found no example.

The following lemma is familiar to some, but is apparently not in the literature.

Lemma. A set of $k-2$ mutually orthogonal latin squares of order $n$ is equivalent to a set of $n^{2}$ ordered $k$-tuples, $\left(a_{i 1}, a_{i 2}, \cdots, a_{i k}\right), i=1, \cdots, n^{2}$, with elements $a_{i j}$ the numbers $1, \cdots, n$, and such that for each pair $u, v$ of distinct numbers from $1, \cdots, k$ and each pair $x, y$ of numbers from $1, \cdots, n$, the relations $a_{i u}=x$ and $a_{i v}=y$ both hold for some $i\left(i=1, \cdots, n^{2}\right)$.

Proof. There being exactly $n^{2}$ ordered $k$-tuples in the set, $a_{i u}=x$ and $a_{i v}=y$ are satisfied for a unique $i$. Associate the $n^{2} k$-tuples with cells of $k-2 n \times n$ matrices, $a_{i 1}$ and $a_{i 2}$ chosen as the row and column indices respectively, and $a_{i j}, j=3, \cdots, k$, the digit in this cell of the $(j-2)$ nd matrix. The conditions on the $k$-tuples imply that the $k-2$ matrices are mutually orthogonal latin squares. For when $u$

Presented to the Society, January 21, 1959; received by the editors February 3, 1959. 
and $v$ are 1 and 2, each cell of the matrices is accounted for. When $u=1$ and $v>2$, each row of the $(v-2)$ nd matrix contains each digit -only once, of course. Similarly when $u=2$ and $v>2$, the same holds on columns. For $u>2$ and $v>2$, each ordered pair of digits occurs (once) in some cell of the $(u-2)$ nd and $(v-2)$ nd matrices. The converse construction of the set of ordered $k$-tuples from the set of mutually orthogonal latin squares is carried out similarly. Since the conditions on the ordered $k$-tuples are symmetric on the positions, any distinct pair $u, v$ from $1, \cdots, k$ may be chosen as row and column indices.

The general result is

THEOREM 1. If there exists a balanced incomplete block design (see [3] for definitions) with $\lambda=1$ and $k$ the order of a projective plane, then there exists a set of $k-2$ mutually orthogonal latin squares of order $v$.

Proof. A projective plane of order $k$ can be represented by a doubly transitive set $S$ of permutations of degree $k$ with the property that for $p$ and $q$ distinct permutations of $S, p q^{-1}$ fixes at most one of the $k$ letters [4]. The class of systems $S$ includes all doubly transitive finite groups, in which only the identity fixes two letters; such groups exist if and only if $k$ is a prime-power [5]. Whether there exists an $S$ of any degree $k$ not a prime-power is equivalent to the unsettled question of existence of a projective plane of order $k$.

Select an ordering of the $k$ digits in each block of the design. Retaining the digits, permute the positions by all elements of a system $S$, thereby generating $k(k-1)$ ordered $k$-tuples from each block. To the class of ordered $k$-tuples already formed, adjoin $(1,1, \cdots, 1)$, $(2,2, \cdots, 2), \cdots,(v, v, \cdots, v)$, each of length $k$. A set of ordered $k$-tuples fulfilling the conditions of the lemma has been constructed.

The above construction is not at all unique; some choices of $v$ and $k$ can be expected to yield a large number of nonisomorphic sets of $k-2$ mutually orthogonal latin squares. First, a balanced incomplete design is not in general determined within isomorphism by its parameters. Also, for $k$ the order of a projective plane, there exist nonisomorphic systems $S$; blocks need not be operated upon by the same $S$. For the Desarguesian plane of prime-power order $k, S$ can be any coset of the doubly transitive group of degree $k$ in which the subgroup fixing a letter is cyclic. Known non-Desarguesian planes determine systems $S$ which are not groups or cosets of groups.

Theorem 1 is specialized and strengthened slightly to yield

Theorem 2. If $m$ is a Mersenne prime $>3$, or $m+1$ is a Fermat 
prime $>3$, then there exists a set of $m$ mutually orthogonal latin squares of order $m^{2}+m+1$. (For all orders included in Theorem 2, the construction of MacNeish produces only $t=2$ orthogonal latin squares.)

Proof. The hypothesis implies that both $m$ and $m+1$ are primepowers. There exists a projective plane of order $m$; that is, a balanced incomplete block design with $\lambda=1, v=m^{2}+m+1$, and $k=m+1$. Also, there exists a projective plane of order $k=m+1$. The hypothesis of Theorem 1 is fulfilled, so that a set of $k-2=m-1$ mutually orthogonal latin squares of order $v$ may be constructed.

For each prime-power $m$, there exists a Desarguesian plane possessing a collineation which is cyclic on all $v$ points [6]. Thus the set of $v^{2} k$-tuples may be constructed so that if $\left(x_{1}, x_{2}, \cdots, x_{k}\right)$ is in the set, then $\left(x_{1}+1, x_{2}+1, \cdots, x_{k}+1\right)$ is also, the addition being modulo $v$. This means that if cell $\left(x_{1}, x_{2}\right)$ of latin square $j-2$ contains digit $x_{j}$, then cell $\left(x_{1}+1, x_{2}+1\right)$ of the same square contains digit $x_{j}+1$-again modulo $v$. Thus, in this restricted situation, there is one more latin square orthogonal to all $m-1$ previously constructed, namely a cyclic square whose $\left(x_{1}, x_{2}\right)$ cell contains $x_{2}-x_{1}+1(\bmod v)$.

In all cases covered by Theorem $2, m \equiv 1(\bmod 3)$. In turn $v=m^{2}+m$ $+1 \equiv 3(\bmod 9)$, so that $t=2$ in MacNeish's construction.

An unfavorable aspect of Theorem 2 is that all orders $v$ of mutually orthogonal latin squares are among those for which Bruck and Ryser [7] have demonstrated nonexistence of projective planes.

Theorem 1 cannot yield the first counter-example to Euler's conjecture of nonexistence of pairs of orthogonal latin squares of orders $\equiv 2(\bmod 4)$. When the order $v$ of the squares is even, the relation $v-1=r(k-1)$ on parameters (with $\lambda=1$ ) of balanced incomplete block designs implies that $r$ is odd and that $k$ is even. In turn, the relation $v r=b k$ yields the information that $k$ is divisible by two to no higher power than is $v$. When $v$ is divisible by two to the first power only, $k$ (necessarily even) is also twice an odd integer. $k=2$ yields no latin squares. Thus a counter-example based on Theorem 1 would require construction in advance of a projective plane of order $\equiv 2(\bmod 4)$, and $>2$, and $a$ fortiori of a counter-example to Euler's conjecture.

The first case where Theorem 2 applies is $m=4$, yielding a set of four mutually orthogonal latin squares of order $4^{2}+4+1=21$. One is the cyclic square, with digit 1 on the principal diagonal. As pointed out in the proof of Theorem 2, the other three latin squares are generated by the rule: if cell $\left(x_{1}, x_{2}\right)$ contains digit $x_{j}$, then cell $\left(x_{1}+1, x_{2}+1\right)$ contains $x_{j}+1$, all $x$ 's modulo 21 . A set of first rows of the three latin squares is given by the following ordered lists: 
$1,7,13,5,12,8,19,21,2,4,14,10,17,20,11,3,16,6,9,15,18$;

$1,19,17,12,10,21,9,18,7,5,20,4,16,15,14,13,3,8,2,11,6$; $1,9,16,10,4,18,2,6,19,12,15,5,3,11,20,17,13,21,7,14,8$.

The author wishes to thank Professor S. K. Stein for informing him that MacNeish had made the conjecture disproved in this paper, and for providing a bibliography [8]; and Dr. Robert Silverman of the Ohio State University for pointing out that in Theorem 1 the system of permutations need not be a group.

\section{REFERENCES}

1. H. F. MacNeish, Euler squares, Ann. of Math. vol. 23 (1921-1922) pp. 221-227.

2. H. B. Mann, The construction of orthogonal latin squares, Ann. Math. Statist. vol. 13 (1942) pp. 418-423.

3. - Analysis and design of experiments, New York, Dover, 1949.

4. Marshall Hall, Jr., Projective planes, Trans. Amer. Math. Soc. vol. 54 (1943) pp. 229-277, Theorem 5.2.

5. W. Burnside, Theory of groups of finite order, 2d ed., Cambridge University Press, 1911; reprinted New York, Dover, 1955, p. 182.

6. James Singer, $A$ theorem in finite projective geometry and some applications to number theory, Trans. Amer. Math. Soc. vol. 43 (1938) pp. 377-385.

7. R. H. Bruck and H. J. Ryser, The non-existence of certain finite projective planes, Canad. J. Math. vol. 1 (1949) pp. 88-93.

8. S. K. Stein, On the foundations of quasigroups, Trans. Amer. Math. Soc. vol. 85 (1957) pp. 228-256.

Remington Rand, UnivaC, Division of Sperry Rand Corporation, St. Paul, Minn. 